\title{
Stochastic Decision-Based Analysis of Admission Control Policy in Multimedia Wireless Networks
}

\author{
Nidal Nasser \\ Department of Computing \& Information Science, \\ University of Guelph, Guelph, Ontario, N1G 2W1 Canada \\ nasser@cis.uoguelph.ca
}

\begin{abstract}
Providing multimedia services with Quality of Service (QoS) guarantees in next generation wireless cellular networks poses great challenges due to the scarce radio bandwidth. Effective Call Admission Control (CAC) is important for the efficient utilization of the limited bandwidth. In this paper, the design of CAC for a decision-based Markovian model of multimedia wireless networks is considered. We propose an optimal CAC policy that aims at maximizing the system utilization while stratifying the QoS constraints bounding the handoff dropping probability of each traffic class. We used the Semi-Markov Decision Process (SMDP) technique to represent the proposed CAC. The optimal CAC decisions for each state are found by solving the linear programming formulation problem. Numerical and Simulation results show that the system upholds the handoff call dropping probability required by each traffic class while maximizing revenue for service providers. The requirements of the mobile users are hence satisfied in periods with different loads, including overload. Moreover, the implemented policy ensures efficient utilization of resources. This latter facet is highly desirable by service providers.
\end{abstract}

\section{Introduction}

With the explosive growth of the wireless communication market, the demand for developing multimedia applications is increasing rapidly. Next generation high-speed Wireless Cellular Networks (WCNs) are expected to support multimedia applications (audio phone, video-on-demand, video-conferencing, Web services, etc.). The third generation (3G) - Universal Mobile Telecommunication System (UMTS) is an example of such a network. Multimedia applications necessitate support of different classes of traffic with diverse Quality of Service (QoS), which need to be guaranteed by WCN. To achieve this goal, QoS provisioning is critical.

One of the main design issues in WCNs is the implementation of Call Admission Control (CAC) policy. The function of the CAC policy is defined as a set of actions to determine if the call request can be accepted or rejected. The condition for accepting a new call request is the availability of sufficient resources (e.g. wireless link bandwidth) to guarantee the QoS requirements without adversely affecting existing calls.

For WCNs, two types of QoS parameters have been introduced which are: New Call Blocking Probability (NCBP) and Handoff Call Dropping Probability (HCDP) 
[1]. The NCBP is the percentage of new calls blocked. New call blocking occurs when all the channels of the wireless medium are busy upon a new call request. The HCDP is the percentage of blocked handoff calls. When a user moves from one cell to a neighboring cell, a handoff takes place. The user requests a channel from the new base station, and if all the channels are busy dropping takes place. Since call dropping of established connections is usually more annoying than rejection of a new connection request, it is widely believed that a wireless network must give higher priority to the handoff connection requests as compared to new connection requests. Therefore, we focus on the handoff dropping probability as the main QoS requirement.

Several CAC policies have been proposed [2], [3], [4] and applied to support identical traffic connections such as guard-channel policy [2]. This policy deals with handoff calls. It reserves a subset of bandwidth allocated to a given cell for handoff calls. Clearly, increasing the number of guard channels will reduce the HCDP and, at the same time, it may increase the NCBP. Berqia et al. [5] has shown that the guardchannel policy is optimal for minimizing a linear objective function of the new and handoff calls. In [6], a learning automaton is used to find the optimal number of guard channels. However, both models assume that the traffic of all connections is identical. This assumption, however, is not valid if multimedia services are to be supported, since multimedia connections may differ in the amount of bandwidth they need to meet their QoS requirements. In order to achieve these requirements, many CAC policies have been presented to support the multimedia services in WCNs [7], [8], [9]. These, however, do not consider the problem of optimizing the admission policy to minimize the probability of dropping a handoff call. In [10], [11], [12], the theory of Semi-Markov Decision Processes (SMDP) is used to construct optimal CAC policies. Yoon et al. [10] proposed a CAC policy using SMPD and used an approximation technique to solve the SMDP-formulated problem. Xiao et al. [11] proposed a genetic algorithm to obtain a near-optimal CAC for multimedia wireless networks and showed some numerical results to illustrate the concept. In [12], Bartolini et al. applied a SMDP formulation to analyze a CAC policy for multiple classes of traffic. A call may transit from one class to another to satisfy the admission requirement. An approximation with iterative solution has been used to obtain the most optimal CAC policy. However, iterative solutions are computationally infeasible for practical size networks. The common disadvantage of the above SMDP models is that they all provide sub-optimal solutions.

In this paper, the theory of SMDP is used to construct an optimal CAC policy for WCNs that support multimedia services. The policy extends and generalizes the guard-channel policy in that it reserves an appreciate amount of bandwidth for different types of classes to assure QoS of handoff calls. We call the modified policy Multi-Class Guard Channel Call Admission Control (MCGC-CAC). Optimal decisions of the MCGC-CAC are obtained by applying SMDP linear programming formulation under hard constraint of the handoff call dropping probability. Our approach is able to achieve optimal performance, maximum bandwidth utilization and guarantee that all handoff calls of different classes of traffic with diverse QoS requirements will experience a dropping probability less than the designated maximum value for each service class. 
The rest of this paper is organized as follows. The network model is presented in Section 2. The multi-class guard channel CAC policy that supports multimedia traffic is proposed in Section 3. The SMDP framework and the optimal CAC policy are presented in Section 4. Numerical results are shown in Section 5. Finally, conclusions drawn from the paper are discussed in Section 6.

\section{Network Model Description}

We consider a network model that supports multiple classes of adaptive multimedia services, which have diverse bandwidth and QoS requirements. We first describe our system model, and then we describe the traffic model.

\subsection{Cell Configuration}

Our approach is based on decomposing the wireless cellular network into individual sub-systems, each corresponding to a single cell. The correlation between these subsystems (models), results from handoff connections between corresponding cells. Therefore, we can model the system at a single-cell level.

Suppose there are K classes of calls in the system (cell). The bandwidth of a classi, i.e., the number of channels required to accommodate the call, is given by $b_{i}$. Let the total bandwidth units (in number of channels) in each cell be the same and denoted by B. The classes are indexed in an increasing order according to their bandwidth requirements, such that:

$$
b_{1} \leq \ldots \leq b_{i} \leq b_{i+1} \leq \ldots \leq b_{K}
$$

In this paper, we assume a Fixed Channel Allocation (FCA) environment. Furthermore, it is assumed that the bandwidth of wideband calls can be scattered across the bandwidth pool as in [9].

\subsection{Traffic Model}

New call arrivals and handoff call arrivals of class-i $(\mathrm{i}=1,2, \ldots, \mathrm{K})$ connections are assumed to follow a Poisson process with rates $\lambda_{n c_{i}}$ and $\lambda_{h_{i}}$, respectively. The total arrival rate of class-i connections is $\lambda_{i}=\lambda_{n c_{i}}+\lambda_{h_{i}}$. The Call Holding Time (CHT) of a class-i call is assumed to follow an exponential distribution with mean $1 / \mu_{b_{i}}$.

For mobility characterization, we assume the following simple model. The Cell Residence Time (CRT), i.e., the amount of time during which a mobile terminal stays in a cell during a single visit, is assumed to follow an exponential distribution with mean $1 / h$ [13]. We assume that the CRT is independent of the service class; hence, connections in any class follow the same CRT distribution. Note that the parameter $h$ represents the call handoff rate.

The channel holding time is the minimum of the CHT and the CRT. As the minimum of two exponentially distributed random variables is also exponentially distributed, then the channel holding time for class-i connection is, therefore, assumed to be exponentially distributed with mean $1 / \mu_{i}$ where $\mu_{i}=\mu_{b_{i}}+h$. Our traffic model is illustrated in Fig. 1. 


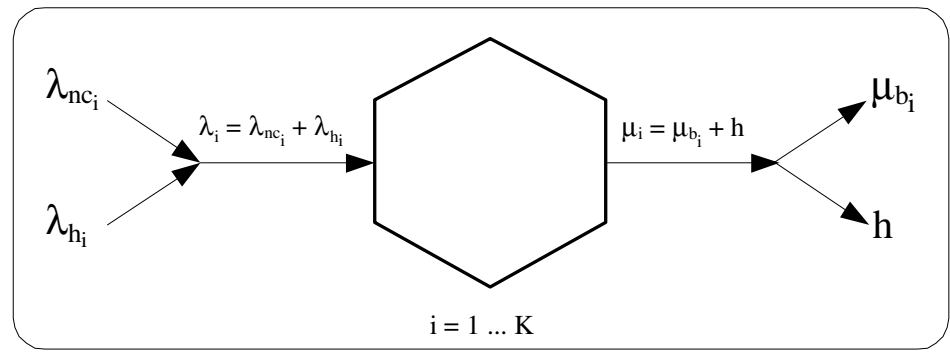

Fig. 1. Traffic model in wireless cellular network

\section{Multi-class Guard Channel Policy}

The proposed Call Admission Control (CAC) policy, Multi-Class Guard Channel (MCGC), is a generalized and enhanced version of the guard-channel policy [2]. The guard-channel policy is modified to be applicable for different traffic classes with different QoS requirements. The principle idea of the existing guard-channel policy is as follows. Considering a single cell with a fixed amount of bandwidth capacity B bandwidth units (in a number of channels), the guard-channel policy gives a higher priority to handoff call requests as opposed to new call requests by reserving a portion of the bandwidth (channels) resource for handoff calls. More specifically, a new call request is admitted only when there are less than $B_{T h}$ channels occupied, where $B_{T h}$ is a threshold between 0 and $B$. on the other hand, a handoff request is rejected only when all $\mathrm{B}$ channels are occupied. As a result, $\mathrm{B}_{\mathrm{R}}=\mathrm{B}-\mathrm{B}_{\mathrm{Th}}$ channels are the guard channels, which are used only by handoff calls.

In this work we generalize the principle idea of the guard-channel policy for the case in which $\mathrm{K}$ separate classes of traffic with diverse bandwidth and QoS requirements exist in the system. Therefore, we design a multi-class guard channel policy that aims to achieve two goals: call prioritization and class differentiation. Similar to the guard-channel policy, our policy reserves a portion of the total bandwidth for handoff calls, thus giving them priority over new calls and potentially providing them with lower handoff call dropping probability. On the other hand, the policy achieves class differentiation as follows. Calls with lower bandwidth requirements will have a better chance at occupying the bandwidth than those with higher bandwidth requirements.

Consider a system with the following parameters:

- $\mathrm{B}_{\mathrm{Th}}$ is the total available bandwidth for different classes of new calls and handoff calls.

- $\mathrm{B}_{\mathrm{R}}=\mathrm{B}-\mathrm{B}_{\mathrm{Th}}$ is the reserved bandwidth for different classes of handoff calls.

- $\mathrm{x}_{\mathrm{i}}$ is the number of ongoing class- $\mathrm{i}$ connections, $\mathrm{i}=1, \ldots, \mathrm{K}$.

- $b_{i}$ is the requested bandwidth of a connection of class-i.

- $\mathrm{b}_{\mathrm{i}, \text { assigned }_{\mathrm{j}}}$ is the assigned bandwidth for call $\mathrm{j}, 1 \leq \mathrm{j} \leq \mathrm{x}_{\mathrm{i}}$, of class- $\mathrm{i}$. 
The modified CAC policy can be described as follows:

Upon the arrival of a new call of class-i, $b_{i}$ \{

$$
\begin{aligned}
& \text { if }\left(b_{i}+\sum_{i=1}^{K} \sum_{j=1}^{x_{i}} b_{\text {, assigned }_{j}} \leq B_{T h}\right) \\
& \text { then accept the call, and } \mathrm{x}_{\mathrm{i}}=\mathrm{x}_{\mathrm{i}}+1
\end{aligned}
$$

else block the call

\}

Upon the arrival of a handoff call of class-i, $b_{i}$

if $\left(b_{i}+\sum_{i=1}^{K} \sum_{j=1}^{x_{i}} b_{i, \text { assigned }_{j}} \leq B\right)$

then accept the call, and $x_{i}=x_{i}+1$

else drop the call

\}

Upon the completion of a call of class-i or handoff to another cell

\{

$$
x_{i}=x_{i}-1
$$

\}

That is, a newly arriving call of class-i will be granted admission if its requested bandwidth, $b_{i}$, plus the current total bandwidth of ongoing connections (new calls and handoff calls) for all classes are less than or equal to $\mathrm{B}_{\mathrm{Th}}$. Whereas, $\mathrm{A}$ handoff arriving call of class- $i$ will be granted admission if its requested bandwidth, $b_{i}$, plus the current total bandwidth of ongoing connections (new calls and handoff calls) for all classes are less than or equal to the total capacity B.

\section{SMDP Formulation}

The aim of this section is to formulate the Multi-Class Guard Channel (MCGC) policy as a Semi-Markov Decision Process (SMDP). Before we proceed an overview of SMDP is given.

\subsection{Overview of SMDP}

The semi-Markov decision process is a stochastic process that describes the evolution of dynamic systems controlled by sequences of decisions or actions. The dynamic system at random discrete time points (epochs) is observed and classified into one of 
a finite number of states. After classification, one of a finite number of possible actions must be chosen and the corresponding revenue for each state is gained due to this decision [14]. For each state $x$, a set of actions is available. If the system is in state $x$ and action $a$ is chosen then:

1. The next state, $y$, of the system is chosen according to the transition probability $p_{x y}^{a}$.

2. The time until the transition from $x$ to $y$ occurs is $\tau(x, a)$.

After the transition occurs, an action is again chosen and (1) and (2) are repeated. Markovian properties are satisfied if at a decision epoch the action $a$ is chosen in the current state $x$, and the state at the next decision epoch depends only on the current state $x$ and the chosen action $a$. They are thus independent of the past history of the system.

\subsection{SMDP Formulation for the Multi-class Guard Channel Policy}

In this section, we first formulate the MCGC policy in a cell as a SMDP. Then the SMDP-formulated problem is represented as a Linear Programming (LP) problem, which aims at both maximizing the bandwidth system utilization while stratifying the QoS constraint to upper bound of the handoff dropping probability.

Whenever there is an arrival of class- $i$ call (new or handoff), CAC must make some decisions. A decision is defined as an action of the system that takes place in a particular state upon a call arrival. The decisions include whether the system accept the call or not. The CAC policy selects its decision from a finite decision (action) space. The possible decision for all kinds of traffic (new call or handoff call) is $\{$ accept/reject $\}$.

The SMDP formulation is characterized by the following 5 ingredients:

\section{1- State Space:}

The current state of the system (cell) can be represented by the vector:

$$
\boldsymbol{x}=\left(x_{1}, x_{2}, \ldots, x_{K}\right)
$$

The non-negative integer $x_{i}$ denotes the number of ongoing new calls and handoff calls class-i connections. Let $\Omega$ denote the state space required to represent our CAC policy. Also, let $\Omega_{B_{T h}} \subset \Omega$ denote the set of states at which a class-i new call and handoff call are accepted, and let $\Omega_{B_{R}} \subset \Omega$ denote the set of states at which a class-i handoff call is only accepted. Thus, according to the multi-class guard channel policy:

$$
\begin{aligned}
& \Omega_{B_{T h}}=\left\{\mathbf{x} \in \Omega \mid 0 \leq \sum_{i=1}^{K} x_{i} b_{i} \leq B_{T h} ; \mathrm{x}_{\mathrm{i}} \geq 0,0 \leq B_{T h} \leq B\right\}, \text { and } \\
& \Omega_{B_{R}}=\left\{\mathbf{x} \in \Omega \mid B_{T h}<\sum_{i=1}^{K} x_{i} b_{i} \leq B ; \mathrm{x}_{\mathrm{i}} \geq 0\right\}
\end{aligned}
$$


Therefore, the state space for the multi-class guard channel policy that contains all admissible states can be represented by:

$$
\Omega=\Omega_{B_{T h}} \cup \Omega_{B_{R}}
$$

A Markov state diagram for the guard-channel policy is shown in Fig. 2 where $\mathrm{B}=6$, $\mathrm{B}_{\mathrm{R}}=3, \mathrm{~b}_{1}=1$ and $\mathrm{b}_{2}=2$.

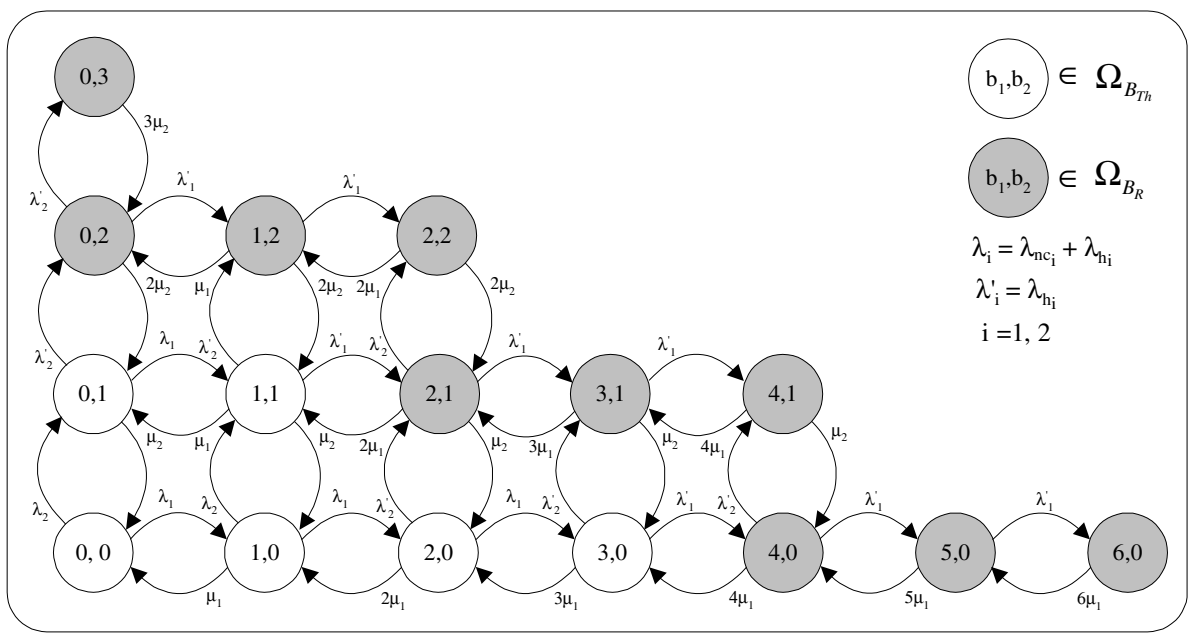

Fig. 2. Markov state diagram for the guard-channel policy with $B=6, B_{R}=3, b_{1}=1$ and $b_{2}=2$

At each state $x$, the CAC policy should select an accept/reject decision for all kind of traffic arrivals, new calls or handoff calls. The set of all the selected decisions are called the admission policy of the system.

\section{2- Decision Epochs and Action Space:}

When an arriving new call or handoff call desires to be admitted into the system, the CAC policy will make a decision as to whether or not to grant admission. We define the decision epochs as the times immediately following the arrival events. A decision epoch is a vector $v=(x, e)$, where $x$ is the vector of class-i calls as in (1) and $e \in\left\{\mathrm{a}_{\mathrm{nc}_{1}}, \ldots, \mathrm{a}_{\mathrm{nc}_{\mathrm{K}}}\right.$, $\left.\mathrm{a}_{\mathrm{h}_{1}}, \ldots, \mathrm{a}_{\mathrm{h}_{\mathrm{K}}}\right\}$. The variable $e$ represents the event type of an arrival and the indicators $\mathrm{a}_{\mathrm{nc}_{\mathrm{i}}}$, and $a_{h_{i}} \in\{0,1\}$ for $\mathrm{i}=1, \ldots, \mathrm{K}$, denote the origination of class-i new call within the cell and the arrival of a class-i call due to handoff from an adjacent cell respectively.

When the system is in state $x$, an accept/reject decision must be made for each type of possible arrival, i.e., an origination of a class-i new call, or the arrival of a class-i handoff call. Thus, the action space A can be expressed as follows:

$$
\mathrm{A}=\left\{\mathrm{a}=\left(\mathrm{a}_{\mathrm{nc}_{1}}, \ldots, \mathrm{a}_{\mathrm{nc}_{\mathrm{K}}}, \mathrm{a}_{\mathrm{h}_{1}}, \ldots, \mathrm{a}_{\mathrm{h}_{\mathrm{K}}}\right): \mathrm{a}_{\mathrm{nc}_{\mathrm{i}}}, \mathrm{a}_{\mathrm{h}_{\mathrm{i}}} \in(0,1), \mathrm{i}=1, \ldots, \mathrm{K}\right\},
$$

where $a_{n_{i}}=0$ (or 1$)$ : reject (or accept) the new call of class-i, and

$a_{h_{i}}=0$ (or 1$)$ : reject (or accept) the handoff call of class-i. 
For example, when $\mathrm{K}=2$ and $\left(\mathrm{a}_{\mathrm{nc}_{1}}, \mathrm{a}_{\mathrm{nc}_{2}}, \mathrm{a}_{\mathrm{h}_{1}}, \mathrm{a}_{\mathrm{h}_{2}}\right)=(0,1,1,1)$ this indicates that only new calls of class -1 will be rejected by the CAC policy.

For a given state $x \in \Omega$, the admissible action space for the system, $\mathrm{A}_{\mathrm{x}} \subset \mathrm{A}$, is defined as follows:

$$
\mathrm{A}_{\mathrm{x}}=\left\{\mathrm{a} \in \mathrm{A}: \mathrm{a}_{\mathrm{nc}_{\mathrm{i}}}=0 \text { and } \mathrm{a}_{\mathrm{h}_{\mathrm{i}}}=0 \text { if } \mathrm{y}=\mathrm{x}+\mathrm{e}_{\mathrm{i}} \notin \Omega, \mathrm{i}=1, \ldots, \mathrm{K}\right\} .
$$

Under this action space, a class-i call is accepted when the state is $x$ if and only if $\mathrm{y}=$ $\mathrm{x}+\mathrm{e}_{\mathrm{i}} \in \Omega$. In other words, $\mathrm{A}_{\mathrm{x}}$ is composed of all those actions in $\mathrm{A}$ that do not result in a transition to a state $\mathrm{y}=\mathrm{x}+\mathrm{e}_{\mathrm{i}} \notin \Omega$. Here $e_{i}$ is a vector of zeros, except for the $\mathrm{i}^{\text {th }}$ component which it is 1 .

\section{3- Expected Sojourn Time:}

If the system is in state $x \in \Omega$ and the action $a \in \mathrm{A}_{\mathrm{x}}$ is chosen, then the expected sojourn time of the state $x, \tau(\mathrm{x}, \mathrm{a})$, is given by:

$$
\tau(\mathrm{x}, \mathrm{a})=\left[\sum_{i=1}^{K}\left(x_{i} \mu_{i}+\lambda_{n c_{i}} a_{n c_{i}}+\lambda_{h_{i}} a_{h_{i}}\right)\right]^{-1},
$$

where $x_{i} \mu_{i}$ represents the rate at which calls terminate within the cell, $\lambda_{n c_{i}} a_{n c_{i}}$ represents the rate at which new calls originate from the cell, and $\lambda_{h_{i}} a_{h_{i}}$ represents the rate of incoming handoffs from adjacent cells to this cell.

\section{4- State Dynamics:}

The state dynamics of a SMDP is completely specified by stating the transition probabilities between the system states. Let $p_{x y}^{a}$ be the transition probability that at the next decision epoch the system will be in state $y$ if the present state is $x$ and the action $a$ is chosen, where $a \in \mathrm{A}_{\mathrm{x}}$. For $y \in \Omega$, we have the following cases:

$$
\begin{array}{lc}
\text { Class-i arrival: } & \mathrm{y}=\mathrm{x}+\mathrm{e}_{\mathrm{i}}: p_{x y}^{a}=\left(\lambda_{n c_{i}} a_{n c_{i}}+\lambda_{h_{i}} a_{h_{i}}\right) \tau(x, a) \\
\text { Class-i departure: } & \mathrm{y}=\mathrm{x}-\mathrm{e}_{\mathrm{i}}: p_{x y}^{a}=\left(x_{i} \mu_{i}\right) \tau(x, a) \\
\text { Otherwise: } & p_{x y}^{a}=0, \quad \forall i \in\{1, \ldots, K\}, x \in \Omega
\end{array}
$$

\section{5- A Revenue Function:}

Let $\mathrm{r}(\mathrm{x}, \mathrm{a})$ be the revenue rate when the cell is in state $x$ and action $a$ is chosen. If $r_{i}$ is the revenue rate of class-i, the total revenue rate for the system is calculated by:

$$
r(x, a)=\sum_{i=1}^{K} r_{i} x_{i}
$$

Assuming that revenue is given by the number of channels assigned, the total revenue rate in state $x$ is equal to the system utilization in state $x$ as follows: 


$$
r(x, a)=\sum_{i=1}^{K} b_{i} x_{i}
$$

where $r_{i}$ is replaced by $b_{i}$ in (9).

\subsection{Constructing Optimal Mutli-class Guard Channel Policy}

SMDPs are usually analyzed and solved within the framework of discrete-time average cost Markov decision processes; see [14] for a detailed discussion. We define decision variable $\pi_{x a}, x \in \Omega$ and $a \in \mathrm{A}_{\mathrm{x}}$, as the long-run fraction of time at which the state $x$ chooses action $a$, and the set of $\pi_{x a}$ collectively determines the CAC policy. Searching for the optimal CAC policy is equivalent finding those decision variables for all states. This can be done by solving the following SMDP Linear Programming (LP) formulation which aims to maximize long-run system utilization and to guarantee QoS for handoff calls.

$$
\begin{aligned}
& \text { Maximize } \sum_{x \in \Omega} \sum_{a \in A_{x}} r(x, a) \tau(x, a) \pi_{x a} \\
& \text { Subject to: } \sum_{x \in \Omega} \sum_{a \in A_{x}} \tau(x, a) \pi_{x a}=1 \\
& \qquad \sum_{a \in A_{x}} \pi_{y a}=\sum_{x \in \Omega} \sum_{a \in A_{x}} p_{x y}^{a} \pi_{x a}, y \in \Omega \\
& \pi_{x a} \geq 0, x \in \Omega, a \in A_{x}
\end{aligned}
$$

The term $\tau(x, a) \pi_{x a}$ in (11) can be interpreted as the long-run fraction of decision epochs when the system in state $x$ and action $a$ is chosen. Hence, the objective function (11) is the system utilization. The constraints are: the normalization condition (12), the global balance equation from the long-run viewpoint (13), and (14) is the constraint on decision variables. The optimal feasible solutions $\pi_{x a}^{*}$ to equation (11) give the optimal CAC policy.

A nice feature of the LP formulation for solving SMDP is that it permits optimization over additional constraints. Since dropping handoff calls is usually less desirable and less tolerable than blocking newly initiated calls, we focus on the handoff dropping probability as the main QoS requirement. Thus, we consider the QoS requirements of the upper bound on the handoff dropping probability of each class-i. Let $d(x, a)=1-a_{h_{i}}$. The stationary handoff call dropping probability for class-i when action $a$ is selected is given by [19]:

$$
P_{d_{i}}=\sum_{x \in \Omega} \sum_{a \in A_{x}} d(x, a) \tau(x, a) \pi_{x a}, i=1, \ldots, K .
$$


We therefore add the QoS constraint of the handoff dropping probability to equation (11) to limit the handoff call dropping probabilities for class-i below a target value $P_{Q o S_{i}}$ :

$$
P_{d_{i}} \leq P_{Q o S_{i}}, i=1, \ldots, K,
$$

where $P_{Q o S_{i}}$ denotes the maximum allowable handoff dropping probability of a class-i.

\section{Numerical Results}

In this section, the performance of the Multi-Class Guard Channel (MCGC-CAC) policy is evaluated using simulation and compared with the Upper-limit (UL) CAC policy [16]. Before proceeding with evaluating the performance of the MCGC-CAC policy, we first describe the simulation model that is used in this paper. Then, we show that the MCGC policy actually achieves its goals in terms of call prioritization and class differentiation. Finally, we show how our formulation to the MCGC-CAC policy as SMDP achieves the optimal solution and outperforms the UL CAC policy, in which it maximizes the bandwidth utilization of the system (cell) and at the same time guarantees QoS for handoff multimedia traffic.

\subsection{Simulation Model}

We simulate one cell with a diameter of $1 \mathrm{~km}$ (i.e., micro cellular environment). The base station resides at the center of the cell. Part of the BS functionalities is the Admission Controller (AC) that operates the CAC policy. The AC components are

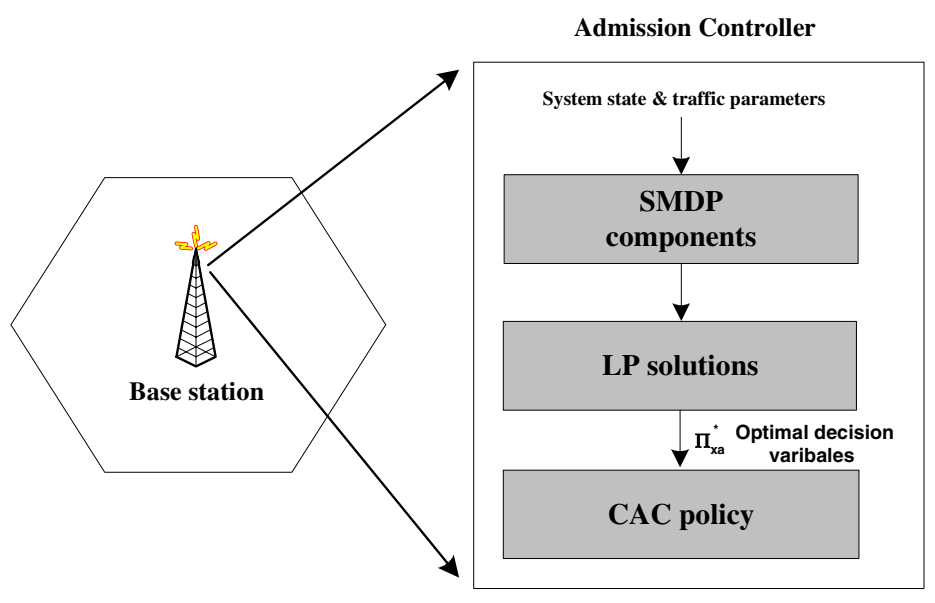

Fig. 3. Admission controller module 
shown in Fig. 3 and operate as follows. Given the current state of the system and the traffic parameters, SMDP components are calculated. Then, the AC uses the LP technique to compute the optimal decision value, $\pi_{x a}^{*}$, that aims at maximizing the system utilization function as given in (11). Solving the LP problem to find the optimal call admission decisions is an offline procedure. That is, the decisions are obtained before invoking the CAC mechanism.

We develop our simulation model based on the following assumptions.

- The total bandwidth capacity of the simulated cell is B basic bandwidth units.

- Two classes of multimedia service are considered. Bandwidth requirement of class-i connection is $b_{i}(i=1,2)$. Class-2 has a higher priority than class- 1 .

- Since class-2 traffic has higher priority than class-1, we assume that the maximum allowable handoff dropping probability for class-2 calls, $P_{Q o S_{2}}$, always has a higher value than $P_{Q o S_{1}}$.

- New call requests of class-i are generated in the cell according to a Poisson process with rate $\lambda_{n c_{i}}$ (calls/second). A newly generated call can randomly appear at any position in the cell with an equal probability. The handoff call arrival rate of class-i is assumed to be proportional to the new call arrival rate of class-i by $\lambda_{h_{i}}=\left(h / \mu_{b_{i}}\right) \lambda_{n c_{i}}$ [17] for $\mathrm{i}=1,2$. Thus, the total arrival rate of class-i calls is $\lambda_{i}=\lambda_{n c_{i}}+\lambda_{h i}$.

- We assume the call holding time of a class-i is exponentially distributed with mean $\mu_{b_{i}}^{-1}$ (seconds). Also, we assume the cell residence time is exponentially distributed with mean $h^{-1}$ (seconds). The channel holding time of class-i calls is exponentially distributed with mean $\mu_{i}^{-1}\left(\mu_{i}=\mu_{b_{i}}+h\right)$.

- Mobiles can travel in one of eight directions with equal probability. A constant randomly selected speed is assigned to a mobile when it enters a cell either at call initiation or after handoff. The speed is obtained from a uniform probability distribution function ranging from $\mathrm{V}_{\min }$ to $\mathrm{V}_{\max }$.

The simulation model is very flexible in which all the above parameters are provided as an input to the simulation program. Thus, this will allow us to test the system with different scenarios. In this paper, we limit our experimental tests to the simulation parameters values that are shown in Table 1 . However, we believe that the higher bandwidth capacity, the more efficiency our policy can achieve since solving the LP problem to find the optimal call admission decisions is an offline procedure. That is, the decisions are found out before the operating of CAC. In addition, techniques for solving large-scale LP problems such as [15], [18] can be applied to the cases of large cellular systems and/or cellular systems with larger capacity. Also, even though there may be some discrepancy between the real bandwidth values of the multimedia service and the values in Table 1 , we believe that our experiments can reflect the real system's behavior.

The performance measures obtained through simulation are New Call Blocking Probability (NCBP), Handoff Call Dropping Probability (HCDP) and bandwidth utilization. 
Table 1. Simulation parameters

\begin{tabular}{|c|c|c|}
\hline Parameter & Value & Unit \\
\hline $\mathrm{B}$ & 30 & $\mathrm{bbu}$ \\
\hline $\mathrm{b}_{1}$ & 1 & $\mathrm{bbu}$ \\
\hline $\mathrm{b}_{2}$ & 2 & $\mathrm{bbu}$ \\
\hline $1 / \mu_{\mathrm{b} 1}=1 / \mu_{\mathrm{b} 2}$ & 500 & $\mathrm{sec}$ \\
\hline $1 / \mathrm{h}$ & 100 & $\mathrm{sec}$ \\
\hline $\mathrm{V}_{\min }$ & 10 & $\mathrm{~km} / \mathrm{hr}$ \\
\hline $\mathrm{V}_{\max }$ & 60 & $\mathrm{~km} / \mathrm{hr}$ \\
\hline
\end{tabular}

\subsection{Performance Evaluation - Relaxed Constraints}

To explore the comprehensive effect of our proposed policy, MCGC, on the NCBP and the HCDP for each traffic class, handoff QoS constraints are relaxed. That is, upper bound of handoff dropping probability of class-i is set to one, i.e., $P_{Q o S_{1}}=$ $P_{Q o S_{2}}=1$. In this experiment, we use the bandwidth threshold, $\mathrm{B}_{\mathrm{Th}}=20$ bandwidth units (channels).

Fig. 4 shows the effect of varying Erlang load on the NCBP and the HCDP of each traffic class. From this figure, we observe that the new call blocking probabilities and the handoff dropping probabilities of both classes increase as the Erlang load increases. However, the HCDP is always lower than the NCBP as a result of the 10 bandwidth units $\left(B_{R}=B-B_{T h}\right)$ reserved exclusively for handoff calls. Moreover, the NCBP and HCDP of class- 1 connections are lower than those of class- 2 connections.

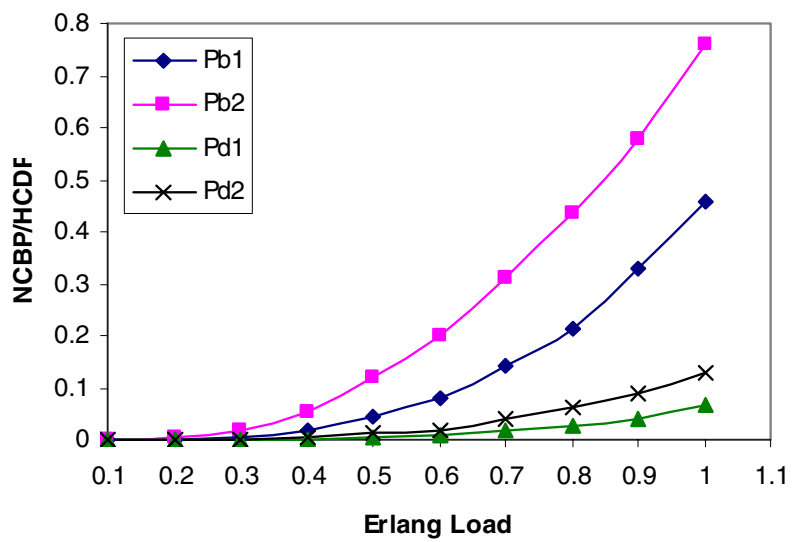

Fig. 4. Effect of varying the Erlang Load on the NCBP and the HCDP

This is because class-1 connections have lower bandwidth requirements, and therefore, they have a better chance in occupying the bandwidth which results in a higher blocking/dropping probability for the higher bandwidth class. The above 
observations show the ability of our policy to prioritize between new calls and handoff calls and to differentiate between traffic classes.

\subsection{Performance Evaluation - Hard Constraints}

In this section, we design several experiments to compare our proposed policy to the UL CAC policy with respect to the HCDP and the bandwidth utilization. The UL CAC policy blocks a new call request of class-i if the number of the calls is greater or equal to an upper-limit value, i.e. threshold $t_{i}$. The UL CAC policy used for comparison has a threshold $t_{1}=10$ and $t_{2}=15$. Simulation parameters are the same as in Table 1 Section 5.1 and $P_{Q o S_{1}}=0.02$ and $P_{Q o S_{2}}=0.04$.

Fig. 5 shows the HCDP for both policies as Erlang load increases. It is shown that the HCDP for the MCGC-CAC policy is bounded by 0.02 and 0.04 for class- 1 and class-2 connections, respectively, and therefore, their QoS requirements are satisfied. On the other hand, the UL CAC policy cannot guarantee such bound, especially when the Erlang load increases.

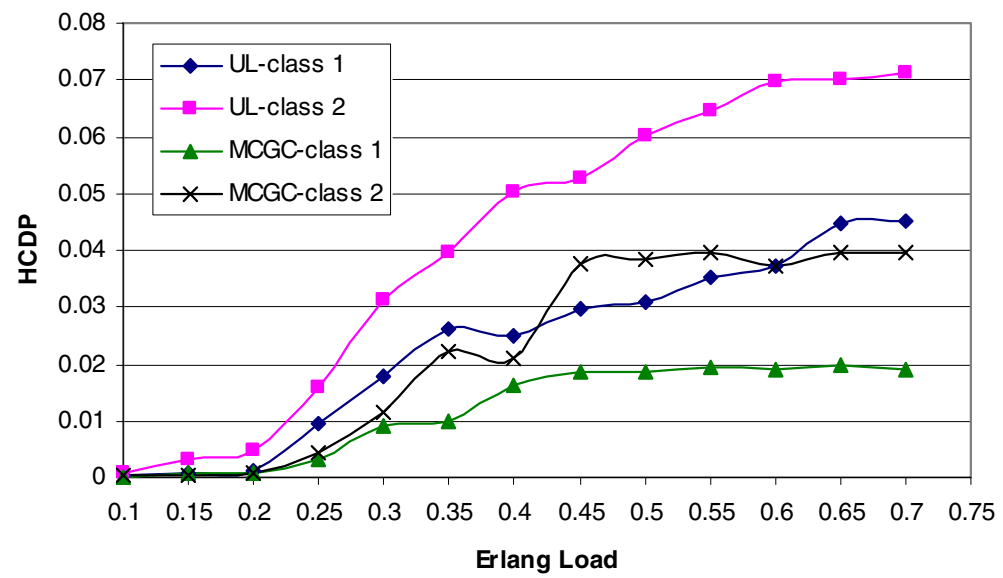

Fig. 5. Handoff call dropping probability vs. Erlang Load

Different cases were investigated as shown in Table 2 to compare between the two policies in term of the bandwidth utilization. The bandwidth utilization of both policies is obtained while the guard channel $B_{R}$ is varying. The last column of Table 2 shows the utilization improvement ratio, UIR, of our policy over the UL CAC policy. The UIR is obtained as follows:

$U I R=\frac{U_{M C G C}-U_{U L}}{U_{U L}} * 100$, where $U_{M C G C}$ is the utilization of the multi-class guard channel policy and $U_{U L}$ is the utilization of the upper-limit policy. 
Table 2. Utilization improvement results

\begin{tabular}{|c|c|c|c|c|c|c|}
\hline No. & $\mathrm{P}_{\mathrm{QoS}_{1}}$ & $\mathrm{P}_{\mathrm{QoS}_{2}}$ & $\mathrm{~B}_{\mathrm{R}}$ & $\mathrm{U}_{\mathrm{MCGC}^{\%}}$ & $\mathrm{U}_{\mathrm{UL}} \%$ & $\mathrm{UIR} \%$ \\
\hline 1 & 0.02 & 0.04 & 5 & $\mathbf{2 9 . 0 8}$ & $\mathbf{2 6 . 8 8}$ & 8.18 \\
\hline 2 & 0.02 & 0.04 & 10 & $\mathbf{2 8 . 1 0}$ & $\mathbf{2 6 . 8 8}$ & 4.52 \\
\hline 3 & 0.02 & 0.04 & 15 & $\mathbf{2 7 . 0 1}$ & $\mathbf{2 6 . 8 8}$ & 0.48 \\
\hline
\end{tabular}

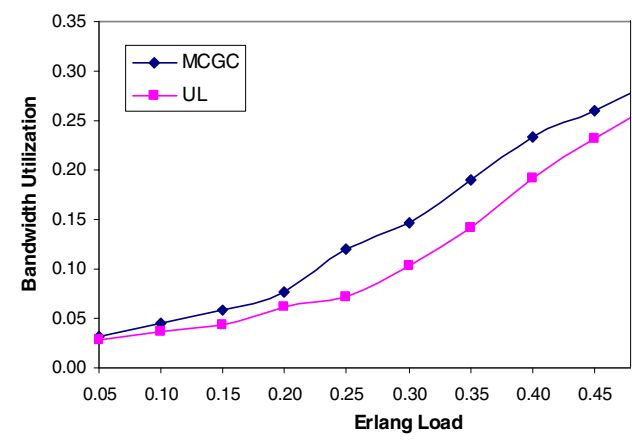

Fig. 6. Bandwidth Utilization vs. Erlang Load $\left(\mathrm{P}_{\mathrm{QoS}_{1}}=2 \%, \mathrm{P}_{\mathrm{QoS}_{2}}=4 \%, \mathrm{~B}_{\mathrm{R}}=5, \mathrm{t}_{1}=10, \mathrm{t}_{2}=15\right)$

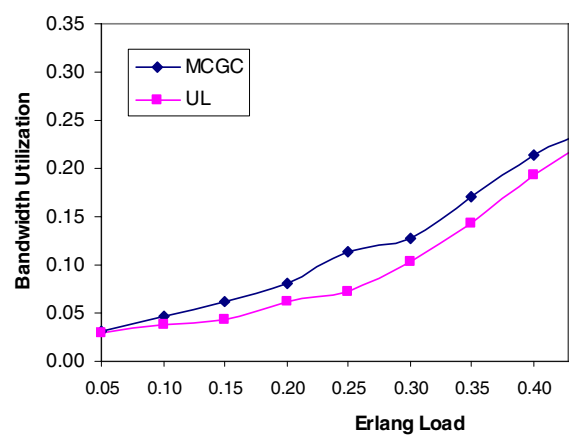

Fig. 7. Bandwidth Utilization vs. Erlang Load $\left(\mathrm{P}_{\mathrm{QoS}_{1}}=2 \%, \mathrm{P}_{\mathrm{QoS}_{2}}=4 \%, \mathrm{~B}_{\mathrm{R}}=10, \mathrm{t}_{1}=\right.$ $\left.10, t_{2}=15\right)$

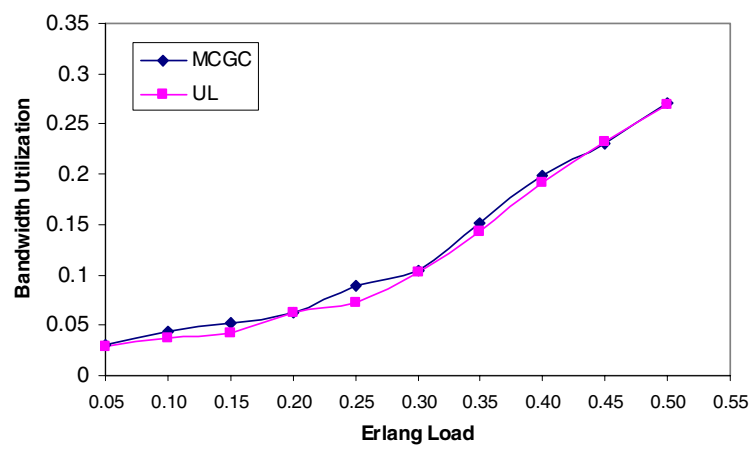

Fig. 8. Bandwidth Utilization vs. Erlang Load $\left(\mathrm{P}_{\mathrm{QoS}_{1}}=2 \%, \mathrm{P}_{\mathrm{QoS}_{2}}=4 \%, \mathrm{~B}_{\mathrm{R}}=15, \mathrm{t}_{1}=10\right.$, $\left.t_{2}=15\right)$

Fig. 6-8 demonstrate the effect of varying the Erlang load on the bandwidth utilization for both policies considering all the cases of Table 2. It is observed that the MCGC-CAC policy (MCGC in the Figures) over SMDP always gets better utilization than the UL CAC policy. Also, we observe that as the reserved bandwidth for 
handoff calls, $B_{R}=B-B_{T h}$ increases, the bandwidth utilization decreases. This is because we are reserving more bandwidth for future handoff calls so that the resource is less utilized. However, the upper bound for the handoff dropping probability is guaranteed which indicates a clear conflict relation between the bandwidth utilization and QoS.

\section{Conclusions}

Providing multimedia services with Quality of Service (QoS) guarantees in next generation high-speed wireless cellular networks poses great challenges due to the scarce radio bandwidth. Effective Call Admission Control (CAC) is important for the efficient utilization of the limited bandwidth. In this paper, we generalized and enhanced the well-known guard-channel policy to accommodate multimedia traffic. Under our new policy, we reserve a fraction of the total available bandwidth in a cell exclusively for multiple classes of handoff calls with each class having distinctively different QoS requirements. The multi-class guard channel CAC policy is formulated as a Semi-Markov Decision Process (SMDP) with constraints on the dropping probabilities of multimedia handoff calls. SMDPs, in the generic sense, are a proven method for evaluation of CAC policies and QoS parameters in wireless networks. In this work, the optimal multi-class guard channel policy decisions are obtained by applying SMDP linear programming formulation. The optimal CAC decisions for each state are found by solving the linear programming formulation with the objectives of maximizing the system utilization and guaranteeing QoS of handoff calls. Simulation results show that the multi-class guard channel policy over SMDP outperforms the upper-limit CAC policy while maximizing the bandwidth utilization and satisfying the upper bound dropping probability of each class of handoff calls.

\section{References}

1. S. S. Rappaport, "The Multiple-Call Hand-off Problem in High-Capacity Cellular Communications Systems", IEEE Transactions on Vehicular Technology, Vol. 40, No. 3, August 1991, pp. 546-557.

2. D. Hong and S. S. Rappaport, "Traffic Model and Performance Analysis for Cellular Mobile Radio Telephone Systems with Prioritized and Non-prioritized Handoffs Procedures”, IEEE Transactions on Vehicular Technology, Vol. 35, No. 3, Aug. 1986, pp. 77-92.

3. S. Choi and K. Shin, "A Comparative Study of Bandwidth Reservation and Admission Control Scheme in QoS-sensitive Cellular Networks", Wireless Networks, Vol.6, 2000, pp.289-305.

4. Y. Iraqi and R. Boutaba, "An Adaptive Distributed Call Admission Control for QoSsensitive Wireless Mobile Networks", Proceedings of the IEEE Wireless Communications and Networking Conference, Chicago, IL, Sept. 2000, pp.449-453.

5. A. Berqia and N. Mikou, "Model and Optimal Call Admission Policy in Cellular Mobile Networks", Springer Lecture Notes in Computer Science, LNCS 1815, October 12, 2001, pp. 920 . 
6. H. Beigy and M. Meybodi, "A Learning Automata Based Dynamic Guard Channel Scheme”, Springer Lecture Notes in Computer Science, LNCS 2510, October 10, 2002, pp. 643.

7. K. Ross and D. Tsang, "The Stochastic Knapsack Problem", IEEE Transactions on Communications, Vol. 37, No. 7, July 1989, pp. 740-747.

8. S. Jordan and P. Varaiya, "Control of Multiple Service, Multiple Resource Communication Networks", IEEE Transactions on Communications, Vol. 42, No. 11, Nov. 1994, pp. 2979-2988.

9. C. Chao and W. Chen, "Connection Admission Control for Mobile Multiple-class Personal Communication Networks", IEEE Journal on Selected Areas in Communications, Vol. 15, No. 8, 1997, pp. 1618- 1626.

10. I. Yoon and B. Lee, "A Distributed Dynamic Call Admission Control that Supports Mobility of Wireless Multimedia Users", Proceedings of the IEEE International Conference on Communications (ICC), 1999, pp. 1442- 1446.

11. Y. Xiao, C. Chen, and Y. Wang, "A Near Optimal Call Admission Control with Genetic Algorithm for Multimedia Services in Wireless/Mobile Networks", Proceedings of the IEEE National Aerospace and Electronics Conference (NAECON), Oct. 2000, pp.787792.

12. N. Bartolini and I. Chlamtac, "Call Admission Control in Wireless Multimedia Networks", Proceedings of the IEEE International Symposium on Personal, Indoor and Mobile Radio Communications (PIMRC), Vol. 1, 2002, pp. 285-289.

13. K. Yeung and S. Nanda, "Channel management in microcell/macrocell cellular radio systems", IEEE Transactions on Vehicular Technology, Vol. 45, Nov. 1996, pp. 601-612.

14. H. Tijms, "Stochastic Modeling and Analysis: A Computational Approach", Wiley, New York, 1986.

15. K. Hastings, "Introduction to the Mathematics of Operations Research", Marcel Dekker Inc., 1989.

16. S. Biswas and B. Sengupta, "Call Admissibility for Multirate Traffic in Wireless ATM Networks", in Proc. IEEE 1NFOCOM, Vol. 2, Kobe, Japan, Apr. 1997, pp. 649-657.

17. N. Nasser and H. Hassanein, "Multi-Class Bandwidth Allocation Policy for 3G Wireless Networks", in Proceedings of the IEEE Conference on Local Computer Networks (LCN), Bonn, Germany, October 2003, pp. 203-209.

18. G.Y. Zhao, "Interior-Point Methods with Decomposition for Solving Large-Scale Linear Programs", Journal of Optimization Theory and Applications, Vol. 102, No. 1, 1999, pp. 169-192.

19. J.M. Hyman, A.L. Lazar and G. Pacifici, "A separation principle between scheduling and admission control for broadband switching," IEEE Journal on Selected Areas in Communications, vol. 11, no. 4, May 1993, pp. 605-616. 